\title{
Coping with COVID
}

The emergence of SARS-CoV-2 and the COVID-19 pandemic have drastically changed life throughout the world.

n mid-December 2019, patients with an unusual form of pneumonia of unknown viral origin began arriving in the hospitals of Wuhan, China. It was quickly established by sequencing the genomic RNA of virus samples that these patients were infected with a novel SARS-like coronavirus, now known as SARS-CoV-2. The full viral RNA genome was released on 10 January 2020. More worrying, however, was that this new virus appeared to be highly infectious. The World Health Organization declared a Public Health Emergency of International Concern on 30 January 2020 and subsequently declared a global pandemic on 11 March 2020.

For reasons that are not yet clear, what is unusual about COVID-19, the disease caused by SARS-CoV-2, is the heterogeneity of both the disease course and immune responses of those infected. Many experience silent or asymptomatic infection, whereas for others, the outcomes can include life-threatening immunopathology, long-term disability, or death. Since the beginning of the pandemic, nearly 110 million cases of viral infection have been confirmed and over 2.3 million deaths due to COVID-19 have been reported.

The public health emergency posed by COVID-19 has led to an unprecedented collaborative global effort, for which the immunology community rightly deserves to be commended, to quickly develop effective vaccines and therapeutics to reduce viral load and limit dysregulated immune responses that contribute to severe disease. Likewise, national governments, with mixed success, have instituted multiple strategies to prevent the introduction of SARS-CoV-2 into their communities, surveillance mechanisms to identify and track viral spread, and efforts to mitigate the spread of the virus once it arrived. Government responses require balancing infection containment measures with socioeconomic concerns, causing friction to arise in multiple locales due to competing interests. Often, the success - or not - of containing SARS-CoV-2 infections is the result of government leaders forming policies based on advice from their public health experts and informed by accurate scientific-based evidence.

We introduce a new series, 'Coping with COVID, for which we have solicited content from immunologists and public health advocates around the globe describing how COVID-19 has affected their country and its response to the SARS-CoV-2 pandemic one-year on. Included in this collection are voices describing how COVID-19 has impacted local research programs and the training of students and post-docs. Throughout the coming year, additional content will be included in this collection, which can be found online at https://www. nature.com/collections/gaacigidef.

In this issue, Le Van Tan describes how Vietnam harnessed its previous experience with the SARS outbreak in 2003 to guide its response to SARS-CoV-2. Although the first human-to-human transmission of SARS-CoV-2 was reported on 23 January 2020 in Vietnam, the country has a surprisingly low infection rate, despite being a nation of over 97 million citizens. This success can be attributed to quickly closing borders to international travelers in February 2020, the institution of rigorous contact tracing measures and minimum quarantine periods for individuals who had direct contact with infected patients, mask requirements to mitigate community spread of the virus and an innovative text messaging program to notify its citizens of potential viral exposures.

Likewise, as Geoghegan et al. report, New Zealand has been largely spared from widespread SARS-CoV-2 infections due to rigorous border control and stringent quarantine measures, including a national lockdown put into place in early March 2020. Despite lacking a prominent biopharmaceutical industry, New Zealand was able to turn to academic laboratories to develop in-house PCR and serology testing kits and genomic sequencing facilities, which proved critical to viral surveillance and contact tracing. Proactive measures were also taken to secure contracts with international biopharmaceutical companies developing COVID-19 vaccines and to partner with neighboring island countries to ensure equitable vaccine access to indigenous peoples.

India is a country of over 1.35 billion. Madkaikar et al. describe the challenges COVID-19 poses to such a large population but also the self-reliance measures that India has implemented since the start of the pandemic. India has launched programs to ramp up indigenous vaccine development, local production of personal protective equipment and diagnostics and antivirals, and has established biorepositories of patient samples. India has also invested in massive public education campaigns to mitigate the spread of SARS-CoV-2.

As reported by Çağdaş, Turkey saw its first COVID-19 case on 11 March 2020, but by the year's end, it had over 2 million confirmed infections, including a large number among healthcare providers, and 18,000 deaths, mostly in elderly populations, similar to other countries. Çağdaş notes the challenges faced by the education system, especially in rural communities, and by postgraduates whose training has been interrupted by the COVID-19 pandemic.

Brazil reported its first COVID-19 case on 25 February 2020, at the height of its Mardi Gras celebration. Since then, Lopes notes that over 9 million infections and 220,000 deaths have occurred. Similar to the US, the anti-science attitudes of government leaders, misinformation sharing via social media and reduced public health funding all contributed to the initial slow response to the COVID-19 pandemic. By contrast, Rabinovich and Geffner report that Argentina's new government restored the Ministry of Science and Technology and implemented an 'all hands on deck' approach to COVID-19. This effort included reorganizing research institutes to direct efforts to the supply of diagnostics, participation in clinical trials of vaccine candidates and antibody-based therapeutics and the establishment of biobanks for primary research endeavors. More impressively, students and young researchers mobilized to form "Anti-Fake News" platforms to combat the spread of false information through social media.

The rapid development of efficacious COVID-19 vaccines has provided hope that a return to 'normal life' may occur within the next year. Yet, viral mutants that can evade vaccine-elicited immunity will continue to pose immediate threats, as will regional disparities in public health care delivery. Thus, it is incumbent on all nations to stay vigilant.

Published online: 24 February 2021 https://doi.org/10.1038/s41590-021-00900-w 